\title{
Glomus tumor of the stomach treated by laparoscopic distal gastrectomy: A case report
}

\author{
TSUTOMU NAMIKAWA $^{1}$, SACHI TSUDA ${ }^{1}$, KAZUNE FUJISAWA $^{1}$, JUN IWABU $^{1}$, \\ SUNAO UEMURA ${ }^{1}$, SHIGEHIRO TSUJII ${ }^{1}$, HIROMICHI MAEDA ${ }^{2}$, HIROYUKI KITAGAWA ${ }^{1}$, \\ MICHIYA KOBAYASHI $^{2,3}$ and KAZUHIRO HANAZAKI ${ }^{1}$ \\ ${ }^{1}$ Department of Surgery, Kochi Medical School; ${ }^{2}$ Cancer Treatment Center, Kochi Medical School Hospital; \\ ${ }^{3}$ Department of Human Health and Medical Sciences, Kochi Medical School, Nankoku, Kochi 783-8505, Japan
}

Received January 25, 2018; Accepted October 19, 2018

DOI: $10.3892 / \mathrm{ol} .2018 .9621$

\begin{abstract}
Glomus tumor of the stomach is a rare submucosal mesenchymal tumor. The present study reports a patient with gastric glomus tumor treated by laparoscopic distal gastrectomy. A 39-year-old male was referred to Kochi Medical School Hospital for examination of a gastric submucosal tumor (SMT) initially diagnosed following a medical check-up. Esophagogastroduodenoscopy revealed a solitary, well-defined, submucosal lesion in the antrum of the stomach. Endoscopic ultrasonography (EUS) revealed a hypoechoic solid mass primarily connected to the gastric muscular layer. Abdominal contrast-enhanced computed tomography confirmed a $1.5 \mathrm{~cm}$, well-defined mass lesion demonstrating homogeneous strong enhancement in the gastric antrum. Subsequent EUS-guided fine-needle aspiration produced a clinical diagnosis of neuroendocrine neoplasm and the patient underwent laparoscopic distal gastrectomy with regional lymph node dissection. Histopathology revealed solid proliferation of round, $\alpha$-smooth muscle actin-immunopositive tumor cells with dilated vessels lined by endothelial cells without atypia, prompting a diagnosis of gastric glomus tumor. To the best of our knowledge, this is the seventh case of gastric glomus tumor treated by laparoscopy reported in English literature. The present case suggested that glomus tumor should be considered in the differential diagnosis for SMT of the stomach.
\end{abstract}

\section{Introduction}

Glomus tumor is a mesenchymal neoplastic proliferation composed of modified smooth muscle cells arising from the

Correspondence to: Dr Tsutomu Namikawa, Department of Surgery, Kochi Medical School, 185-1 Kohasu, Oko-cho, Nankoku, Kochi 783-8505, Japan

E-mail: tsutomun@kochi-u.ac.jp

Key words: glomus tumor, laparoscopic gastrectomy, endoscopic ultrasonography, fine needle aspiration biopsy perivascular structure called a glomus body (1). This tumor occurs most commonly in the peripheral soft tissues and extremities, and is seldom found in internal organs (2). Gastric glomus tumors are rare, with an estimated frequency of approximately $1 \%$ that of gastrointestinal stromal tumors (GISTs) $(3,4)$.

In this report, we present a case of gastric glomus tumor in a 39-year-old male who was treated by a laparoscopic distal gastrectomy. The clinical characteristics of previously reported cases are also discussed.

\section{Case Report}

A 39-year-old Japanese man was referred to our hospital for further examination of gastric submucosal tumor (SMT) diagnosed during a medical check-up. His past medical history and family history were unremarkable. The laboratory findings were almost within normal limits, as were serum levels of carcinoembryonic antigen and cancer antigen 19-9. Esophagogastroduodenoscopy (EGD) revealed a SMT of approximately $2 \mathrm{~cm}$ in size in the greater curvature side of the antrum (Fig. 1). Endoscopic ultrasonography (EUS) demonstrated a hypoechoic solid mass lesion with a small anechoic component in the third or fourth tissues layer, suggesting a lesion in the muscular layer of the stomach (Fig. 2).

Abdominal contrast-enhanced computed tomography revealed a $1.5-\mathrm{cm}$, well-defined mass lesion in the gastric antrum with strong, homogeneous enhancement in the early (Fig. 3). To obtain a definite diagnosis, EUS-guided fine-needle aspiration (EUS-FNA) was performed using a 22-gauge needle. The cytopathology smear of the lesion obtained by EUS-FNA revealed the proliferation of small, round to oval tumor cells with round nuclei and scant cytoplasm (Fig. 4). The patient underwent laparoscopic distal gastrectomy with reginal lymph node dissection under a clinical diagnosis of neuroendocrine neoplasm (NEN).

Gross examination of the surgically resected specimen showed a SMT (Fig. 5A, arrow) that appeared in cross-section as a well-circumscribed, solid tumor measuring $1.5 \times 1.0 \mathrm{~cm}$ (Fig. 5B). Microscopical examination reveals solid proliferation of tumor cells with oval-shaped nuclei and scanty cytoplasm around the disorganized vessels in the submucosa and the muscular layer (Fig. 6). Immunohistochemical staining showed 


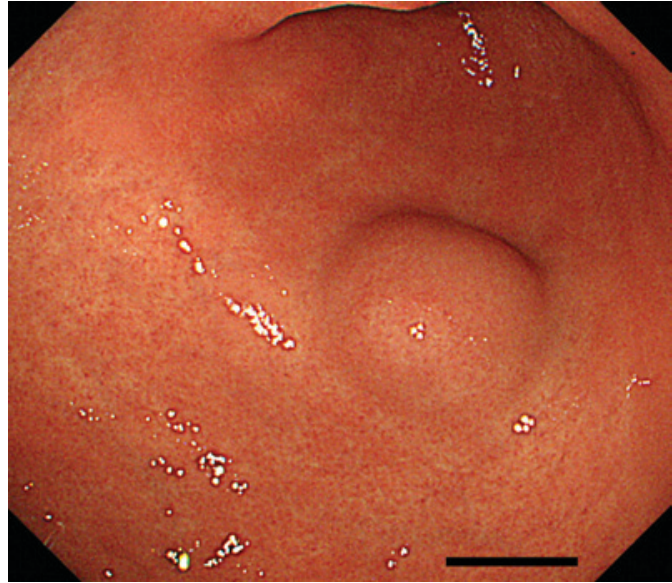

Figure 1. Esophagogastroduodenoscopy demonstrating a submucosal tumor on the greater curvature of the antrum. Scale bar, $1 \mathrm{~cm}$.

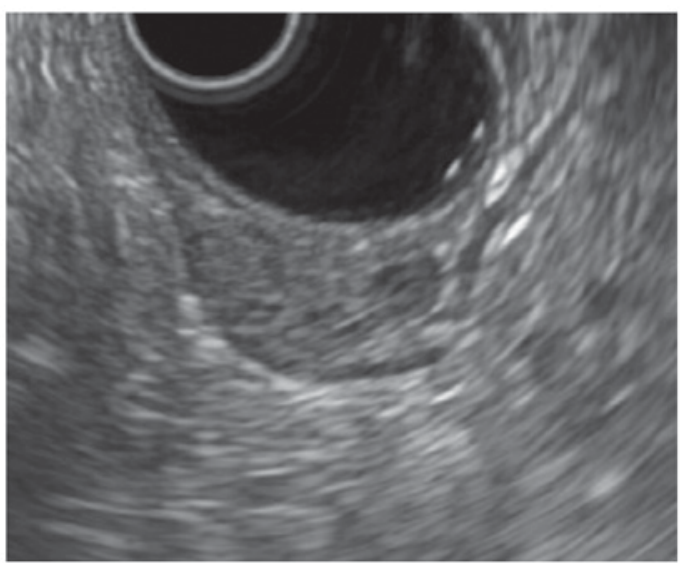

Figure 2. Endoscopic ultrasonography showing a heterogeneous hypoechoic mass in the muscularis propria without evidence of deep involvement.

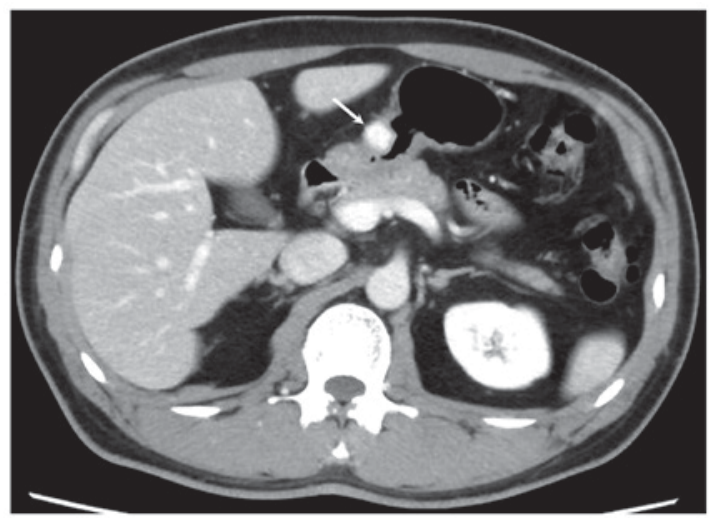

Figure 3. Abdominal contrast-enhanced computed tomography showing a well-defined mass lesion with homogeneous strong enhancement in the gastric antrum, measuring $1.5 \mathrm{~cm}$ in diameter (white arrow).

a positive signal for $\alpha$-smooth muscle actin, but no staining for desmin, c-kit, CD34, S-100, chromogranin A, synaptophysin, and CD56. Approximately $1 \%$ of the cells were positive for the proliferation marker Ki-67. Therefore, we diagnosed a glomus tumor of the stomach. The postoperative course was uneventful, and he was discharged on postoperative day 10 .

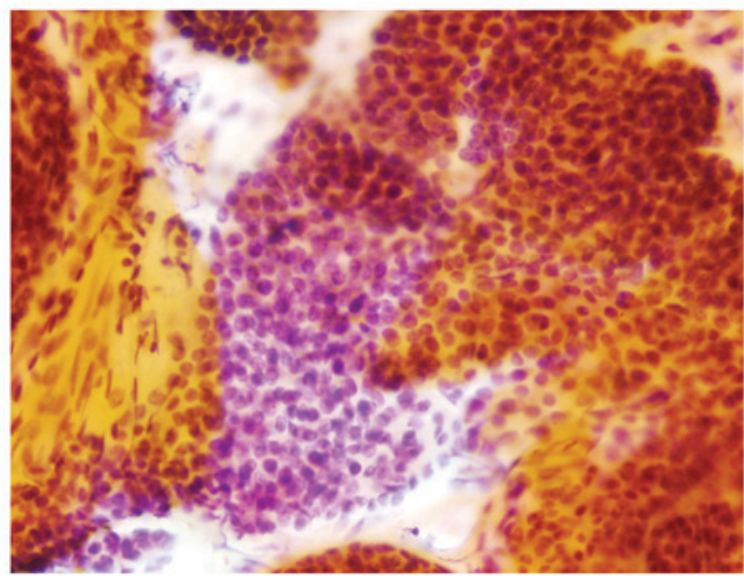

Figure 4. Giemsa staining of cytological smear obtained by endoscopic ultrasonography-guided fine-needle aspiration showed solid clusters of tumor cells with round nuclei and scant cytoplasm. Magnification, x200.

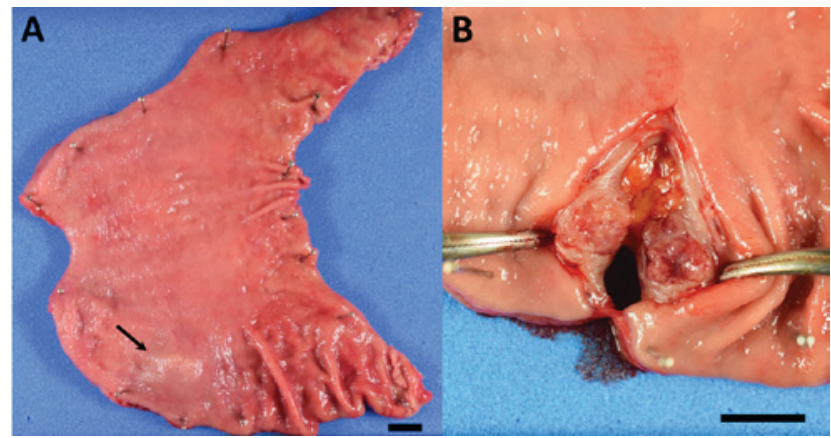

Figure 5. (A) Gross examination of the surgically resected specimen showed a submucosal tumor within an intact mucosa (black arrow). (B) The mass was a well-circumscribed, $1.5 \mathrm{~cm}$ diameter solid tumor. Scale bar, $1 \mathrm{~cm}$.

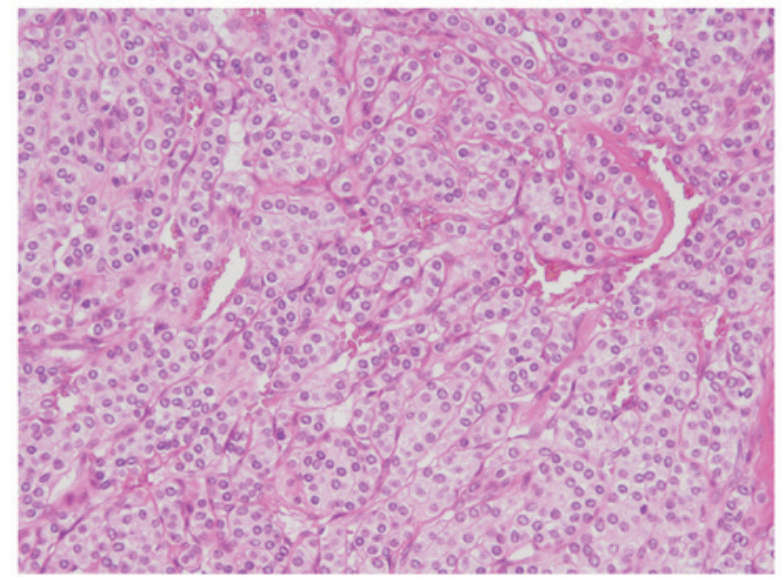

Figure 6. Histological findings of the resected specimen demonstrating well-circumscribed solid nests of round tumor cells next to slit-shaped vessels. Magnification, x200.

\section{Discussion}

Herein we describe a rare case of glomus tumor of the stomach treated by laparoscopic distal gastrectomy. In preparing this report, we searched for English-language articles on similar cases published from 2000 to 2016 in the Medline and PubMed 
Table I. Clinicopathological data for reported cases of gastric glomus tumor treated laparoscopically.

\begin{tabular}{|c|c|c|c|c|c|c|}
\hline Author & Age (years) & Sex & Tumor location & Tumor diameter $(\mathrm{cm})$ & Treatment & (Refs.) \\
\hline Minoda et al & 50 & Female & L, Ant & 1.2 & LWR & (3) \\
\hline Halawani et al & 33 & Female & L, Gre & 1.6 & LDG & (5) \\
\hline Zhao et al & 51 & Female & M, Less & 2 & LWR & (6) \\
\hline Kato et al & 52 & Male & L, Gre & 3.5 & LECS & (7) \\
\hline Castro et al & 70 & Female & L, Gre & 2.5 & LWR & (8) \\
\hline Ebi et al & 45 & Female & L, Less & 1.5 & NEWS & (1) \\
\hline Present case & 39 & Male & L, Gre & 0.8 & LDG & \\
\hline
\end{tabular}

L, Lower third of the stomach; M, Middle third of the stomach; Less, lesser curvature; Gre, greater curvature; Ant, anterior wall; LECS, laparoscopy and endoscopy cooperative surgery; NEWS, non-exposed endoscopic wall-inversion surgery; LWR, laparoscopic wedge resection; LDG, laparoscopic distal gastrectomy.

databases, using the keywords 'glomus tumor', 'stomach', and 'laparoscopy' to obtain data on age, gender, tumor location, tumor size, depth of invasion, histological type, treatment, and outcome for each patient. To the best of our knowledge, this is only the seventh reported case of a gastric glomus tumor treated laparoscopically in the English literature.

Table I lists clinicopathological features of the six previously reported cases $(1,3,5-8)$ and the present case. The median patient age was 50 (range 33-70 years), and the male-to-female ratio was 2:5. Gastric tumor in the lower third of the stomach was reported in six cases, with the remaining patient showing a lesion in the middle-third of the stomach. Treatment consisted of laparoscopic wedge resection in three patients, laparoscopic distal gastrectomy in two patients, laparoscopy and endoscopy cooperative surgery (LECS) in one patient, and non-exposed endoscopic wall inversion surgery in the remaining patient. The median tumor size was $1.6 \mathrm{~cm}$ (range $0.8-3.5 \mathrm{~cm}$ ).

As gastric glomus tumor lacks specific clinical and endoscopic characteristics, it is difficult to distinguish them from the more common GIST or NEN including carcinoid tumor by conventional imaging $(9,10)$. EUS-FNA is an effective method for obtaining pathological specimens of gastric SMT with a high rate of diagnostic accuracy (7). In the present case, the Giemsa staining of cytological smear obtained by EUS-FNA showed solid clusters of tumor cells with round nuclei and scant cytoplasm. On the basis of these histological findings, a preoperative diagnosis of a gastric NEN was made. However, because NEN comprises oval or spindle tumor cells arranged in cords or nests with thin-walled blood vessels, they can be extremely difficult to distinguish from glomus tumor. Consequently, a definitive diagnosis of gastric glomus tumors cannot be confirmed by standard endoscopy until histological results including immunohistochemical examination are considered $(11,12)$. If the immunohistochemical staining of the specimens were performed, taking into the possibility of glomus tumor consideration, an accurate preoperative diagnosis might have been possible.

Complete resection of the lesion is necessary for accurate characterization and to treat the patient appropriately due to the possibility of malignant transformation, even though most glomus tumors are benign (11). Such efforts to treat SMT of the stomach have been improved by recent advances in endoscopic technique and devices that allow not only laparoscopic resection including LECS and non-exposed endoscopic wall-inversion surgery (NEWS), but also endoscopic resection $(11,13)$.

In conclusion, although gastric glomus tumor is extremely rare, physicians should take this disease into consideration especially when SMT is observed in the lower third of the stomach. To avoid overtreatment of the glomus tumor, every effort including EUS-FNA and immunohistochemical examination must be carried out to make accurate diagnosis. Further studies and assessments by the accumulation of additional cases are needed to establish precise diagnostic criteria and to distinguish other diseases presenting as SMT of the stomach.

\section{Acknowledgements}

Not applicable.

\section{Funding}

No funding was received.

\section{Availability of data and materials}

The datasets during and/or analysed during the current study available from the corresponding author on reasonable request.

\section{Authors' contributions}

TN and MK designed the study. TN, ST, KF, JI, SU, ST, HM, $\mathrm{HK}, \mathrm{MK}$ and $\mathrm{KH}$ acquired, analyzed and interpreted the data within the study. TN and $\mathrm{KH}$ finalized the manuscript and submitted the paper for publication. All authors edited the manuscript for intellectual content. All authors read and approved the final manuscript.

\section{Ethics approval and consent to participate}

Not applicable. 


\section{Patient consent for publication}

Written informed consent was obtained from the patient for publication of this case report and any accompanying images.

\section{Competing interests}

The authors declare that they have no competing interests.

\section{References}

1. Ebi M, Sugiyama T, Yamamoto K, Saito T, Inoue T, Yamaguchi Y, Tamura Y, Izawa S, Hijikata Y, Funaki Y, et al: A gastric glomus tumor resected using non-exposed endoscopic wall-inversion surgery. Clin J Gastroenterol 10: 508-513, 2017.

2. Yamagata S, Shoji T, Kawashima S, Narasaka T, Sato S, Kawamura M, Matoba $\mathrm{N}$ and Wakasa $\mathrm{H}$ : Glomus tumor of the stomach. Tohoku J Exp Med 78: 202-208, 1962.

3. Minoda Y, Akahoshi K, Oya M, Kubokawa M, Motomura Y and Nakamura K: Gastric glomus tumor diagnosed by endoscopic ultrasound-guided fine-needle aspiration biopsy: Report of a case. Fukuoka Igaku Zasshi 105: 105-109, 2014.

4. Miettinen M, Paal E, Lasota J and Sobin LH: Gastrointestinal glomus tumors: A clinicopathologic, immunohistochemical, and molecular genetic study of 32 cases. Am J Surg Pathol 26: 301-311, 2002

5. Halawani HM, Khalife M, Safadi B, Rida K, Boulos F and Khalifeh F: Laparoscopic antral resection with Billroth I reconstruction for a gastric glomus tumor. Int J Surg Case Rep 5: $1128-1131,2014$
6. Zhao XJ, Wang HH, Sheng JQ and Li N: Laparoscopic resection of a gastric glomangioma. Endoscopy 46 (Suppl 1 UCTN): E73-E74, 2014

7. Kato S, Kikuchi K, Chinen K, Murakami T and Kunishima F: Diagnostic utility of endoscopic ultrasound-guided fine-needle aspiration biopsy for glomus tumor of the stomach. World J Gastroenterol 21: 7052-7058, 2015.

8. Castro Ruiz C, Carlinfante G, Zizzo M, Giunta A, Ronzoni R, Azzolini F and Pedrazzoli C: Glomus tumor of the stomach: GI image. J Gastrointest Surg 21: 1099-1101, 2017

9. Lin YM, Chiu NC, Li AF, Liu CA, Chou YH and Chiou YY: Unusual gastric tumors and tumor-like lesions: Radiological with pathological correlation and literature review. World J Gastroenterol 23: 2493-2504, 2017.

10. Papadelis A, Brooks CJ and Albaran RG: Gastric glomus tumor. J Surg Case Rep 2016: pii: rjw183, 2016.

11. Zhang Y, Zhou P, Xu M, Chen W, Li Q, Ji Y and Yao L: Endoscopic diagnosis and treatment of gastric glomus tumors. Gastrointest Endosc 73: 371-375, 2011.

12. Baek YH, Choi SR, Lee BE and Kim GH: Gastric glomus tumor: Analysis of endosonographic characteristics and computed tomographic findings. Dig Endosc 25: 80-83, 2013.

13. Namikawa T and Hanazaki K: Laparoscopic endoscopic cooperative surgery as a minimally invasive treatment for gastric submucosal tumor. World J Gastrointest Endosc 7: 1150-1156, 2015. 